\title{
Heavy Fuel Oil Droplet Breakup and Dispersion Study
}

\author{
Masoud Darbandi ${ }^{1}$, Ali Fatin ${ }^{1}$, Gerry E. Schneider ${ }^{2}$ \\ ${ }^{1}$ Department of Aerospace Engineering, Centre of Excellence in Aerospace Systems, Sharif University of Technology \\ P. O. Box 11365-11155, Tehran, Iran \\ darbandi@sharif.edu; afatin4@gmail.com \\ ${ }^{2}$ Department of Mechanical and Mechatronics Engineering, University of Waterloo \\ Waterloo, Ontario, Canada \\ gerry.schneider@uwaterloo.ca
}

\begin{abstract}
The impact of flow swirl on heavy fuel oil (HFO) droplet breakup and dispersion is investigated using the finite-volume method. The numerical framework considers suitable models to predict the droplets' breakups and their dispersions affected by their interaction with turbulence. The validation of chosen models is carried out by comparing the current results with those of previous numerical studies. After validation procedure, three different flow conditions are constructed to expand the study to various swirl number influences. The aim is to investigate the interaction of HFO spraying with the crossed axial swirling flow. The results show that the droplet Sauter mean diameter decreases continuously and the spray becomes finer as the swirling flow strength increases. Dispersion of the finer spray grows up and the spray becomes wider for the stronger swirling flow conditions. Also, the focus on the droplets' concentration shows that the droplets' concentration decreases for higher swirl number flows. This reduction is mainly due to the droplets' dispersion into a larger volume of space. In other words, the spray's width becomes much wider by increasing the swirling influences. It can be concluded that the strong swirling flow can improve the combustion quality of HFO via improving the mixing process and also via controlling the flame structure. The quantifications are performed subsequently.
\end{abstract}

Keywords: Heavy Fuel Oil, Swirling Flow, Droplet Breakup, Sauter Mean Diameter (SMD), Dispersion

\section{Introduction}

Heavy fuel oil (HFO) is still an important source of energy especially in developing countries. Its combustion investigation is considered as a very challenging field of research due to its very specific characteristics. It is because HFO has very complex combustion chemistry. It needs fuel spray, the droplets breakup and dispersion, evaporation, fast mixing, and proper ignition. Among the above demands, the good droplet breakup and dispersion can have significant impact on the improvement of other processes because it can eventually result in perfect combustion conditions with suitable heat transfer and possibly lower $\mathrm{NO}_{\mathrm{x}}$ emission. Up to today, lots of valuable researches have been launched to recognize the spray breakup and dispersion in detail for various liquids; using both experimental and numerical methods. Some experimental studies have been presented in past publications. Hiroyasu and Kadota [1] investigated a high speed liquid jet of diesel fuel in an enclosed cylindrical apparatus filled with constant temperature $(293 \mathrm{~K})$ quiescent nitrogen. The spray penetration length and the droplet Sauter mean diameter (SMD) were measured at various pressures of chamber. Liu and Reitz [2] investigated the air-assist liquid drop atomization process. Their experiment resulted in useful information about the microscopic structure of the liquid breakup process, drop breakup regimes, drag coefficient of drops, drop size distribution, and drop trajectories. Wu et al. [3] experimentally investigated the structures of spray plumes of water jet injected into a subsonic crossflow. They measured the droplet size, the axial velocity, and the volume flux at various axial distances. They studied the liquid/air momentum flux ratio effect on the spray penetration, spray widths, and spray crosssectional area behaviours. Becker and Hassa [4] investigated the kerosene fuel breakup in crossflow experimentally. They derived the correlations for the jet penetration and the lateral dispersion. Besides the past experimental research works, the numerical simulations have been also fulfilled to put comment on the spray breakup and dispersion. In addition to such experimental approaches, we can find some important numerical studies in this issue. For example, Liu et al. [5] evaluated different spray models using the measured data reported by Ref. [2]. They found that the drag coefficient of drop and the breakup time model constant would be adjusted to match the measurements. Tanner [6] investigated the atomization process of a liquid fuel jet numerically. He developed a secondary breakup model in his study and tested it using some 
measured data. Barroso et al. [7] studied the sensitivity of results to mesh resolution and structure numerically. They attempted to quantify these sensitivities and to verify the computational results on the basis of detailed shadowgraphy and PDA experiments for a constant volume bomb. Behzad et al. [7] modelled the liquid jet breakup and dispersion in crossflow. They compared their results; including the droplet's size, velocity, and volume flux distributions, with the available experiments, which performed in similar flow conditions. In addition to the above pure experimental and numerical studies, the HFO spray characteristics effect on combustion has been also investigated both experimentally and numerically. For example, Ballester and Dopazo [8] experimentally investigated the influence of atomization characteristics on the HFO combustion. Two different atomization cases were tested under various combusting conditions. They compared their spatial distributions including the velocity field, temperature and species characteristics, with the measured data. Takei et al. [9] presented the results of atomization using the correlated model of HFO combustion in an industrial furnace. They proposed a specific simulation framework for their HFO combustion simulations.

Generally, the NOx concentrations depend on the flame structure, which can be also affected significantly by the spray breakup and dispersion behaviours. On the other hand, many industrial burners use swirling air to stabilize and control the flame. Therefore, the interactions between swirling air and HFO droplets have always been a challenging subject for the combustion researchers. There are some experimental efforts to address this important issue [10-12]. These references investigated and reported their particular spray configuration and its swirling air effect. Unfortunately, none of the previous mentioned works focused on the HFO spray and its interaction with the nearby swirling air. Indeed, the numerical methods have been rarely used in this topic by past researches. So, this paper investigates the flow swirl impact on the HFO droplet breakup and dispersion just numerically. First, the CFD method is validated for the droplets and swirling air interaction against the measurements reported in Ref. [2]. To quantify the swirling effect on the droplets breakup and dispersion correctly, three different test cases are simulated and the results are presented and discussed for their droplets paths, SMD, and spray widths.

\section{Mathematical Modelling}

In present study, an Eulerian-Lagrangian approach is used to investigate the interactions between the swirled flow and incoming droplets benefiting from suitable the droplets-turbulence interaction and the droplets secondary breakup modelings. In this regard, the continuous phase equations are solved via Eulerian approach. On the other hand, the liquid droplets, as the dispersed phase, are tracked individually within the flow field in a Lagrangian frame. The standard k- $\varepsilon$ turbulence model is used to solve the transport equations for the turbulent flow. Similar to the experiments of Ref. [2], it is assumed that the air flow and the liquid fuel are at the same room temperature and there is no thermal energy transfer to the surrounding domain. So, the evaporation is not considered and the droplets are modelled as inert particles in the flow. This section provides the dispersed phase equations and the model for the interaction between turbulence and droplets. Also, it presents the effect of secondary breakup model used in present study in details.

\subsection{Dispersed Phase Equations}

As known, the instantaneous motion and trajectory of droplets are modelled using the Lagrangian form of the governing equations. Reference [13] presents the axial, radial, and tangential momentum equations for each droplet. For example, the momentum equation in the $x$-direction can be written as

$$
\frac{d u_{d}}{d t}=\frac{3 \bar{\rho}}{4 \rho_{d} D} C_{D}\left(u-u_{d}\right)\left|\mathbf{u}-\mathbf{u}_{\mathbf{d}}\right|
$$

where $u_{d}$ and $\mathrm{u}$ represent the local droplet and the gas velocity, respectively. In Eq. (1), the drag force effect is only considered and the gravity and other body force effects are neglected accordingly. Equation (1) calculates the drag force acting on a droplet with a diameter of $\mathrm{D}$ and a density of $\rho_{d}$; considering a drag coefficient of $C_{D}$. The relative speed between the gas and droplet would cause such drag forces, which play main role in the droplet breakup process.

To determine the droplet drag coefficient, we use the dynamic drag model. This model takes into account the impact of droplet distortion on the drag coefficient. In this model, the value of drag coefficient is varied between the case of a rigid sphere (with no distortion) to a disk (with a maximum distortion) [5]. The drag coefficient is then calculated from 


$$
C_{D}=C_{D, \text { sphere }}(1+2.635 y)
$$

where $y$ is the drop distortion. Equation (2) states that the drag coefficient varies linearly between that of a sphere and a value of 1.54 corresponding to a disk case [5].

The turbulent fluctuations of gas phase can have significant effects on the droplet dispersion behaviour. To predict the droplet dispersion, we use the turbulence stochastic tracking (random walk) model. Hence, the trajectory of droplets is predicted using the instantaneous value of the fluctuating gas velocity $(u)$ in Eq. (1). Thus, we have

$$
u=\bar{u}+u^{\prime}
$$

To predict the instantaneous gas velocity, we use the discrete random walk model. This model simulates the interaction of a particle with a succession of discrete stylized fluid phase turbulent eddies [13].

\subsection{Heavy Fuel Oil Droplet Breakup}

To calculate the droplet breakup, we use the Taylor analogy breakup (TAB) model, which considers a liquid drop to be analogous to a spring-mass system. In this model, the droplet breakup is due to an increase in the amplitude of the droplet oscillations up to a critical value. Indeed, if the drop distortion (y), which was introduced in Eq. (2), exceeds unity the parent droplet will break up into a number of smaller child droplets. The size of the resulting child droplets is determined by considering the energy balance between the parent droplet and the child droplets. The number of child droplets is calculated from the determined size for the child droplets considering the mass conservation equation. More details for TAB breakup model can be found in O'Rourke and Amsden [14].

\section{Computational Model}

\subsection{Droplet Breakup and Dispersion Simulation Validation}

Before examining the air swirl impact on the droplets breakup and dispersion, it is necessary to validate the chosen numerical models. In this regard, the test case and the test conditions chosen by Ref. [2] are selected to evaluate the current numerical results with the experimental data reported by Liu and Rietz [2] and the available numerical solutions reported by Liu et al. [5].

The experiments of Liu and Rietz [2] were carried out for nine different cases with various Weber numbers. However, the approximate weber number is about 36 in various practical HFO firing systems. Since this value is very close to the weber number of case 2 in the above experimental investigations, case 2 is selected as the reference test case to verify the results of present numerical simulations. In this test case, the non-swirling air jet flow has a (vertical) velocity of $59 \mathrm{~m} / \mathrm{s}$ when it comes into the ambient. To avoid the droplet vaporization, all tests were performed in ordinary room temperature. Also, the mono-disperse stream of liquid fuel drops is generated using an injector with an injection diameter of $170 \mu \mathrm{m}$ with a (horizontal) velocity of $16 \mathrm{~m} / \mathrm{s}$. The physical properties of the utilized fuel include a density of $824 \mathrm{~kg} / \mathrm{m}^{3}$, the dynamic viscosity of $2.17 \times 10^{-3} \mathrm{~Pa} . \mathrm{s}$, and a surface tension coefficient of $0.02 \mathrm{~kg} / \mathrm{s}^{2}$. If required, more information on fuel and the injection system can be found in Ref. [2].

The current simulations are carried out using the numerical models introduced in Section 2. The general droplets trajectory is illustrated Fig. 1. As shown in this figure, the current droplets path agrees well with the path illustrated by Ref. [2]. Additionally, Fig. 2 presents the spatial variation of SMD of spray. As seen, the SMD is about $76 \mu \mathrm{m}$ in experiment at the end of breakup process, i.e., $x=10 \mathrm{~mm}$. However, the predicted SMD is about $47 \mu \mathrm{m}$ at this distance. It means that it is $38 \%$ less than that of measurement. Also, comparing the present simulation results with the data taken from Liu simulation, some conclusion can be drained. Figure 2 indicates that despite good agreement of the current SMD prediction with those of Liu simulation at the end of droplet breakup process, there are some deviations in the predicted SMDs at the earlier distances. Such deviations can be attributed to the weakness of droplet-turbulence interaction model chosen in the present study. It may also be attributed to the use of different time steps to calculate the droplets motion. 


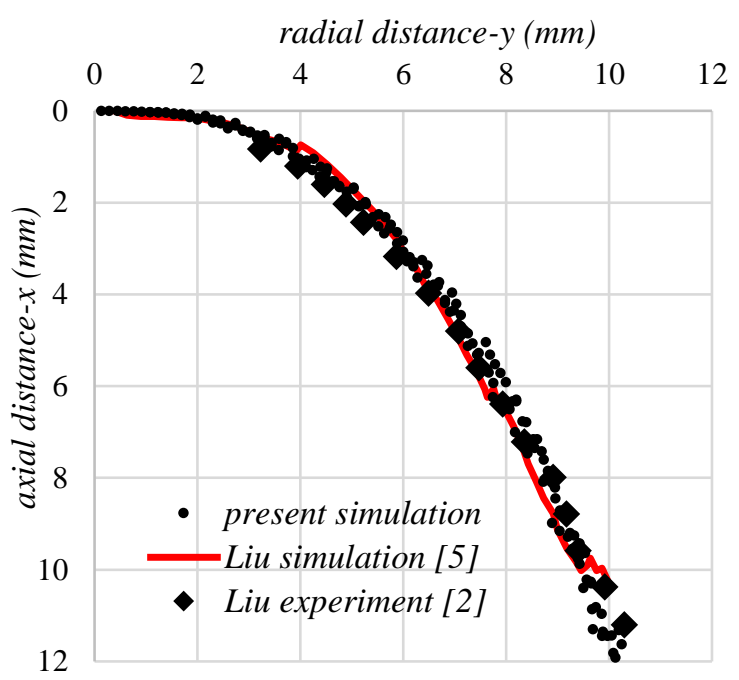

Fig. 1: The current droplets trajectory path and comparisons with those of other numerical solutions and experimentals.

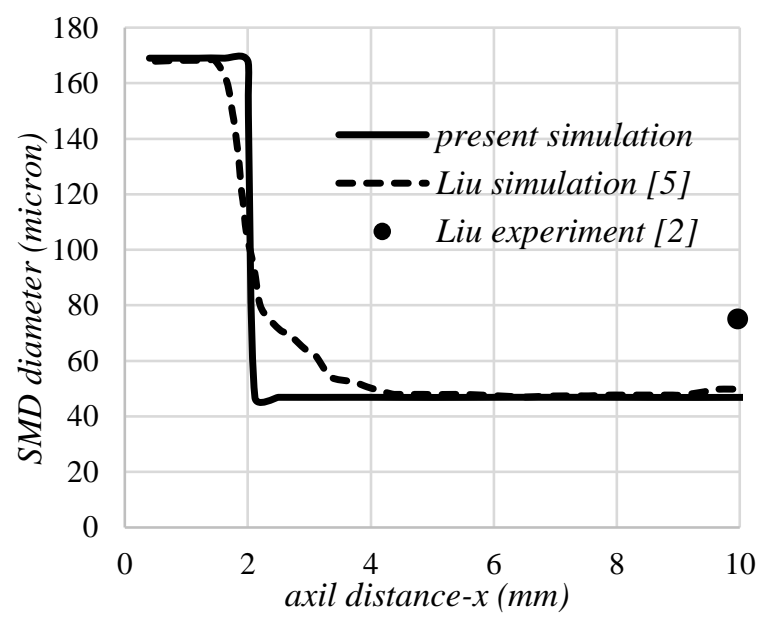

Fig. 2: The current spatial variation of SMD diameters and comparisons with those of other numerical solutions and experimentals.

\subsection{Computational Setup and Boundary Conditions}

The computational domain, which is used to study the flow swirl impact on the HFO droplet breakup and dispersion, is the same as that we used to validate the results of preceding simulations. However, the type of fuel is changed to a heavy fuel oil one. The physical specifications for the current HFO include the density of $990 \mathrm{~kg} / \mathrm{m}^{3}$, the dynamic viscosity of $3.57 \times 10^{-2} \mathrm{~Pa}$. s, and the surface tension coefficient of $0.028 \mathrm{~kg} / \mathrm{s}^{2}$. Also the inlet air is suitably swirled at the flow inlet. The flow swirl is characterized by its swirl number value, which is defined as the ratio of the axial flux of angular momentum to the axial flux of axial momentum [15]. Table 1 introduces three cases with three different air swirl numbers, which are required to be applied at the air inlet. In all cases, the axial air velocity is constant and equal to $59 \mathrm{~m} / \mathrm{s}$; however, the tangential velocity is suitably changed to achieve the desired target swirl numbers.

Table 1: The air swirl numbers for the three chosen test cases.

\begin{tabular}{|l|c|}
\hline \multicolumn{1}{|c|}{ Case } & Swirl Number \\
\hline Case 1 & 0.6 \\
\hline Case 2 & 1 \\
\hline Case 3 & 1.3 \\
\hline
\end{tabular}


The computations are carried out in a three-dimensional cylindrical domain having a diameter of $52 \mathrm{~mm}$ and a length of $57 \mathrm{~mm}$. A total number of 193,000 cells is created and refined in the zone, where the HFO droplets are conducted into the air jet. The utilized mesh and boundary conditions are shown in Fig. 3 schematically. Figure 3 also shows the applied wall, inlet, and outlet boundary conditions. The gas phase is assumed to be steady and the droplets are tracked within time choosing a time-step of $10 \mu \mathrm{s}$. The finite-volume method is used to discretize the governing equations. The pressure field is calculated using the SIMPLE algorithm. The second-order upwind discretization scheme is used to model the convection terms in the Navier-Stokes equations.

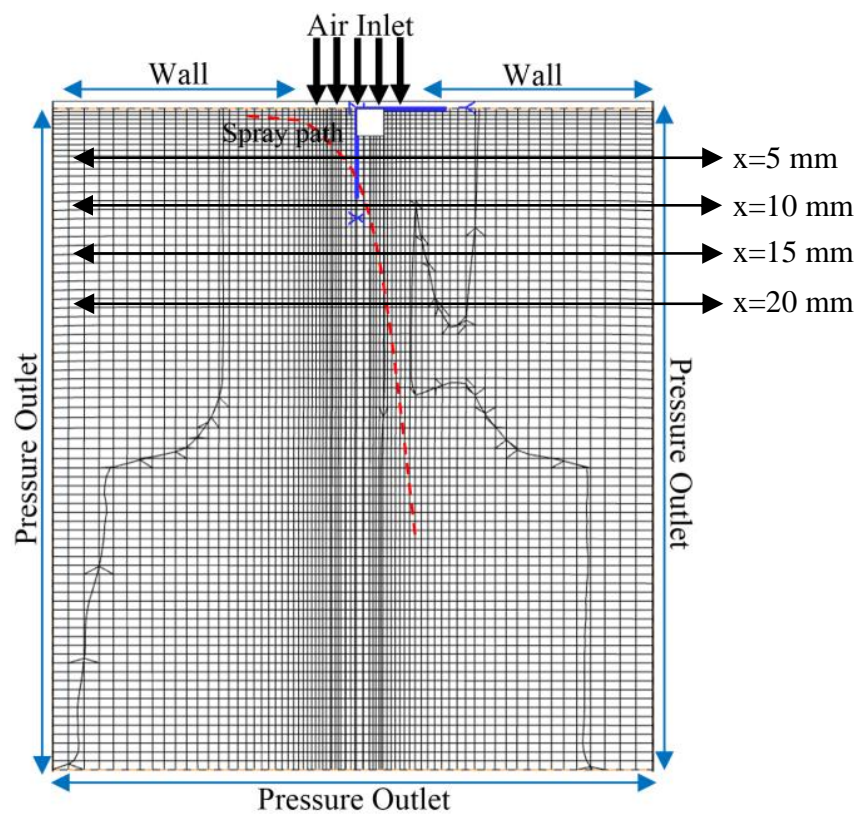

Fig. 3: Schematic of the current 3D mesh and the applied boundary conditions.

\section{Results and Discussion}

To evaluate the swirl impact on the HFO droplet's breakup and dispersion, we investigate the spray parameters including the SMD, the droplet's path and the spray width magnitudes. The results are presented accordingly in three parts.

\subsection{Flow Swirl Impact on Droplet Breakup}

The SMD diameter is assumed to be a good representative of fuel spray fineness. A lower SMD diameter means that the parent droplets have been broken up into smaller droplets and it accordingly generates a finer spray. To investigate the swirl impact on the droplet breakup, we simulate the three test cases introduced in Table 1. Figure 4 presents the spatial distribution of SMD diameter along the mean flow direction, i.e., $x$-direction. Comparing the three produced distributions with each other, it can be recognized that the breakup process is almost completed at $x=10 \mathrm{~mm}$. Increasing the flow swirl number from 0.6 to 1.3 , the SMD diameter decreases continuously. In other words, the spray becomes much finer. Also, Fig. 4 shows that at the earlier steps of breakup processes, say between $x=2$ to $3 \mathrm{~mm}$, the breakup process grows faster for higher swirl number values and the breakup length of droplets becomes shorter in flows with higher swirl number magnitudes. 


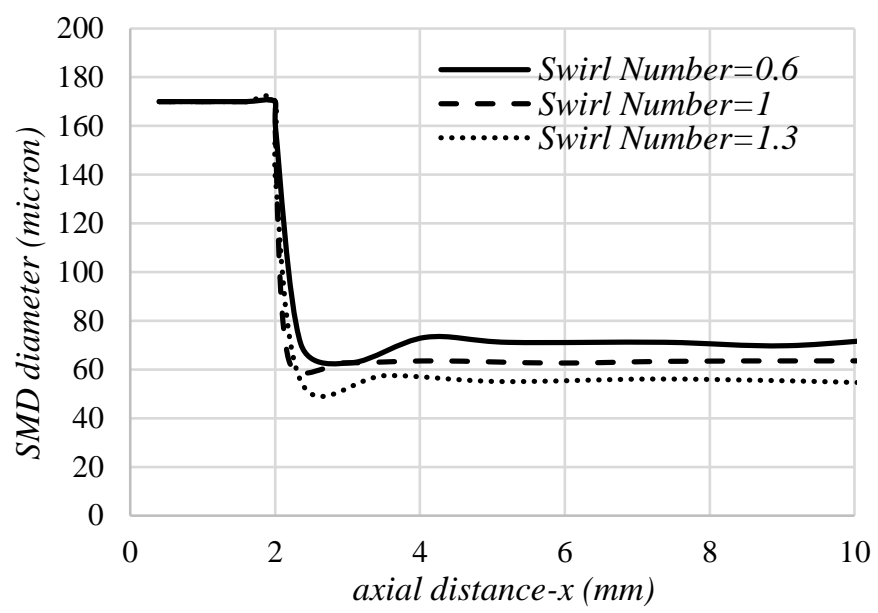

Fig. 4: Spatial variation of SMD considering various swirl numbers.

\subsection{Flow Swirl Impact on Droplets Path}

Figure 5 depicts the droplets path in $x-y$ plane considering different swirl number magnitudes. The black, blue, red, and green circles show the droplets path for the swirl numbers of $0.0,0.6,1.0$, and 1.3 , respectively. It can be seen that the spray has the minimum deflection for a swirl number of zero. However, the spray has the maximum deflection for the swirl number of 1.3. Therefore, it can be said that the spray path deflects more by increasing the swirl number. This is because the weber number increases as the flow becomes more swirled. As was concluded in Fig. 4, this would result in smaller droplet sizes. On the other hand, the smaller droplets are affected more by the momentum of continuous phase and this makes the spray to become more deflected toward the air flow direction in such conditions.

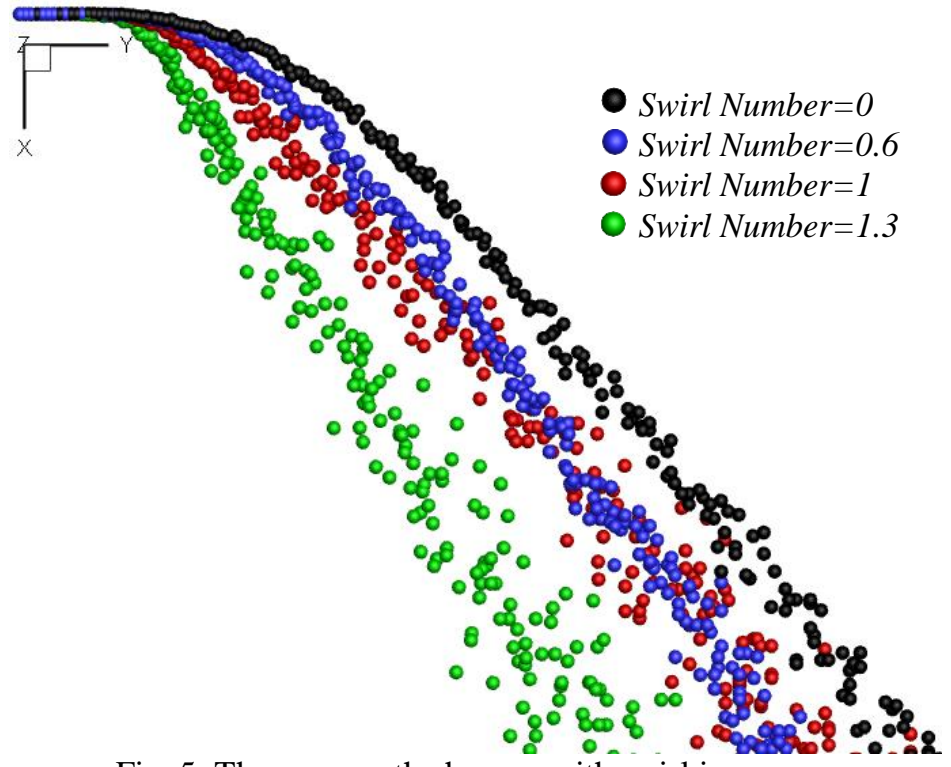

Fig. 5: The spray path changes with swirl increases.

Figure 6 shows the droplets path in the $y$-z plane considering the previous swirl number values. In this figure, the red apheres represents the tangential velocity direction. The black spheres show the droplets dispersion for the zero swirl number magnitude. In this case, the droplets have not dispersed well and the spray width would be apparently minimum. By increasing the swirl number from zero to 0.6, the spray's width increases. Also, the figure shows that there are more serious spray deflections for the cases with swirl numbers of 1.0 and 1.3. However, in spite a stronger spray deflection for the case with a swirl number of 1.3, its spray width is close to the case with a swirl number of 1.0. Achieving a finer spray 
condition at higher swirl number values, it can be concluded that the small droplets have been affected more by the air flow. So, the turbulence dispersion would be more effective there.

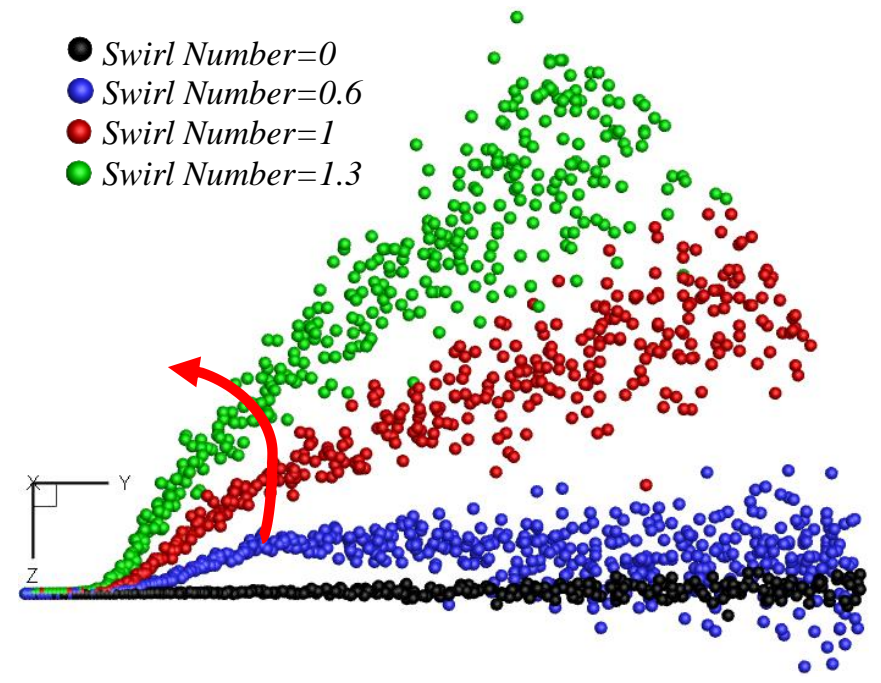

Fig. 6: The droplets dispersion (spray width) for flows with different swirling strengths.

\subsection{Flow Swirl Impact on Spray Width}

It is quite normal to have more reduction in the droplets concentration when the spray penetrates more in the $x$ direction because of its influences in a larger volume of space. So, it is recommended to consider the droplets concentration distribution as a good criterion to evaluate the droplets dispersion and subsequently the spray's width. Figure 7 illustrates the droplets concentration distribution in the radial direction at four different axial distances. These axial locations are shown in Figure 3 by four horizontal black lines. Here, we present the results for all the three chosen test cases introduced in Table 1, i.e., the swirl numbers of 0.6, 1.0, and 1.3. The distributions for these three cases are shown in Figs. 7(a), 7(b), and 7(c), respectively. As is expected, the droplets concentration is higher at distances closer to the injector, e.g., $x=5 \mathrm{~mm}$; however, it decreases substantially in farther distances, e.g., $x=20 \mathrm{~mm}$. For example, the droplets concentration is about $11 \mathrm{~kg} / \mathrm{m}^{3}$ at $\mathrm{x}=5 \mathrm{~mm}$ for a swirl number of 0.6 (see Fig. 7(a)); however, it decreases to about 1.0 $\mathrm{kg} / \mathrm{m}^{3}$ at $\mathrm{x}=20 \mathrm{~mm}$. Figure 7(b) shows the results for a swirl number of 1.0. As seen, the maximum value of droplets concentration reduces to $5.5 \mathrm{~kg} / \mathrm{m}^{3}$ at $x=5 \mathrm{~mm}$, while it is about $0.6 \mathrm{~kg} / \mathrm{m}^{3}$ at $x=20 \mathrm{~mm}$. The variation is similarly observed for a swirl number of 1.3; however, with a sharper reduction. Figure 7(c) shows that the maximum droplets concentration is about $4 \mathrm{~kg} / \mathrm{m}^{3}$ at $x=5 \mathrm{~mm}$, however, it reduces to $0.5 \mathrm{~kg} / \mathrm{m} 3$ at $x=20 \mathrm{~mm}$. Generally speaking, the droplets concentration reduces more effectively in higher swirling flows. In other words, the droplets dispersion is stronger for higher swirling flow cases.

Comparing the sprays' widths in Figs. 7(a) to 7(c) with each other, one can conclude that the spray's width would be higher for the swirl number of 1.3. This can be readily inferred by inspecting the distributions and their peaks at farther axial distances, say $x=20 \mathrm{~mm}$. On the other hand, one can claim that the penetration depth would be lower for this swirl number because of a higher amount of the accompanying airflow momentums. 
a)

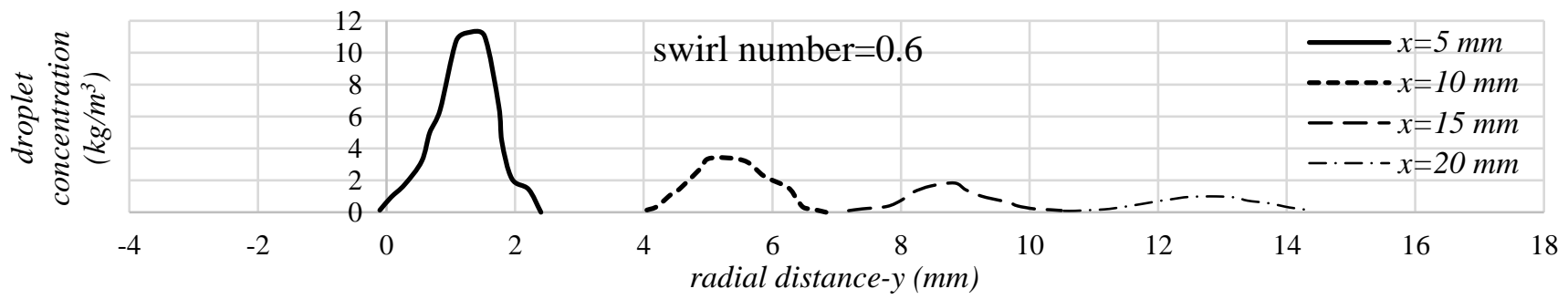

b)

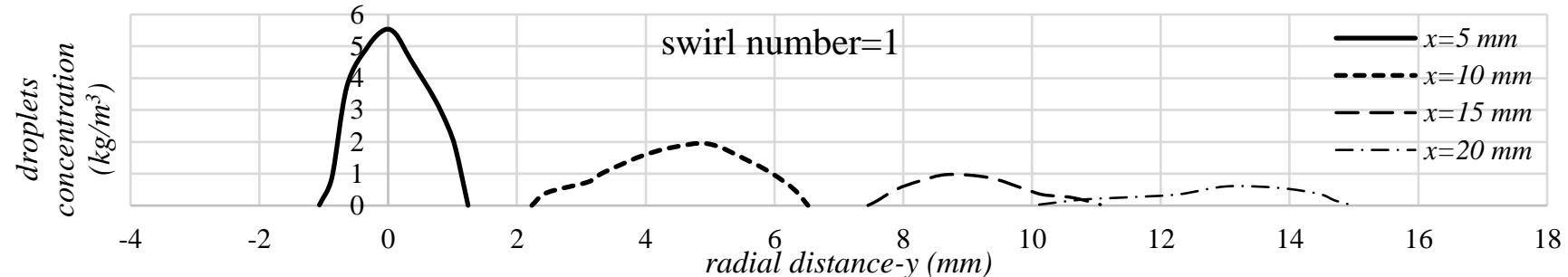

c)

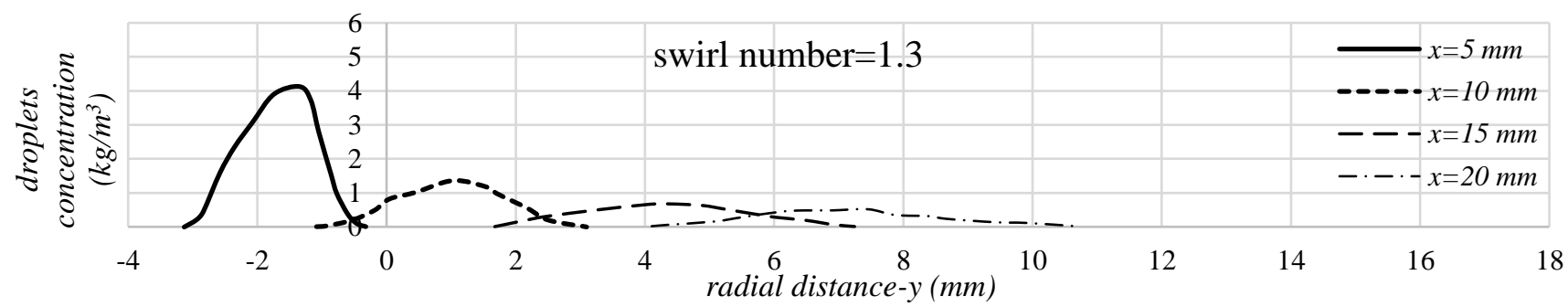

Fig. 7: The droplets concentration distribution in radial direction $(y)$ for various axial distances $(x)$; a) swirl number of 0.6 , b) swirl number of 1.0, and c) swirl number of 1.3.

\section{Conclusions}

A numerical simulation framework was used to investigate the flow swirl impact on the HFO spray dispersion. Before evaluating the interaction between the swirled flow and the HFO spray, it was necessary to validate the utilized numerical simulation framework. This was done by comparing the current solutions with those of previous experimental and numerical results. The numerical simulation of HFO spray was then carried out considering various air flow conditions with differing air swirling flow strengths. The results showed that the SMD diameter of spray would become smaller and the spray would become finer as the swirling flow became stronger. On the other hand, by decreasing the SMD diameter, due to increasing the swirl number, the droplets were affected more seriously by the momentum of continuous phase. Therefore, the droplets obeyed the air flow path more effectively and they resulted in higher spray deflection magnitudes. Also, the droplets concentration investigation showed that the droplets concentrations would decrease for stronger swirling conditions. This reduction was mainly due to the dispersion of droplets in larger volume contents. In other words, the spray's width became wider with increasing the airflow swirl. This increase can be mainly attributed to the turbulence dispersion impact, which shows stronger influences on finer droplets. Also, the droplets concentration investigation showed that the penetration depth for the spray would reduce for more serious swirling flows. Additionally, the results of this research showed that the swirl of air would play critical role in the quality of HFO spray and subsequently its combustion efficiency. Indeed, there were sensitive impacts on the spray fineness and droplet dispersion. Therefore, one can suitably control the HFO flame shape by suitable airflow swirling.

\section{Acknowledgements}

The authors gratefully acknowledge the financial funds received from the Deputy of Research and Technology in Sharif University of Technology.

\section{References}

[1] H. Hiroyasu and T. Kadota, "Fuel droplet size distribution in diesel combustion chamber," Bulletin of JSME, vol. 19, no. 135, pp. 1064-1072, 1976. 
[2] A. Liu and R. Reitz, "Mechanism of air-assisted liquid atomization," Atomization and Sprays, vol. 3, no. 1, pp. 5575, 1993.

[3] P. Wu, K. Kirkendall, R. Fuller and A. Nejad, "Spray structures of liquid jets atomized in subsonic crossflows," Journal of Propulsion and Power, vol. 14, no. 2, pp. 173-182, 1998.

[4] J. Becker and C. Hassa, "Breakup and atomization of a kerosene jet in crossflow at elevated pressure," Atomization and Sprays, vol. 12, no. 1-3, pp. 49-68, 2002.

[5] A. Liu, D. Mather and R. Reitz, "Modeling the effects of drop drag and breakup on fuel sprays," SAE Technical Paper, SAE, 1993.

[6] F. Tanner, "Liquid jet atomization and droplet breakup modeling of non-evaporating diesel fuel sprays," SAE Technical Paper 970050, SAE, 1997.

[7] M. Behzad, A. Mashayek, and N. Ashgriz. "A KIVA-based model for liquid jet in crossflow," In ILASS Americas, 22nd Annual Conference on Liquid Atomization and Spray Systems. 2010.

[8] J. Ballester and C. Dopazo, "Experimental study of the influence of atomization characteristics on the combustion of heavy oil," Combustion Science and Technology, vol. 103, no. 1-6, pp. 235-263, 1994.

[9] M. Takei, R. Weber and T. Niioka, "Mathematical modeling of industrial furnaces considering detailed oil spray characteristics," Combustion Science and Technology, vol. 175, no. 7, pp. 1237-1262, 2003.

[10] B. Rho, S. Kang, J. Oh and S. Lee, "Swirl effect on the spray characteristics of a twin-fluid jet," KSME International Journal, vol. 12, no. 5, pp. 899-906, 1998.

[11] R. Hadef and B. Lenze, "Effects of co- and counter-swirl on the droplet characteristics in a spray flame," Chemical Engineering and Processing: Process Intensification, vol. 47, no. 12, pp. 2209-2217, 2008.

[12] K. Rajamanickam and S. Basu, "Insights into the dynamics of spray-swirl interactions," Journal of Fluid Mechanics, vol. 810, pp. 82-126, 2016.

[13] A. Gosman and E. Loannides, "Aspects of computer simulation of liquid-fuelled combustors," Journal of Energy, vol. 7 , no. 6 , pp. 482-490, 1983.

[14] P. O'Rourke and A. Amsden, "The TAB method for numerical calculation of spray droplet breakup," SAE Technical Paper 872089, SAE, 1987.

[15] A. Gupta, D. Lilley and N. Syred, Swirl flows, 1st ed. Tunbridge Wells: Abacus, 1985. 